\title{
Maximum Likelihood Estimation with Poisson (Counting) Statistics for Waste Drum Inspection
}

\author{
D. Goodman
}

May 1997

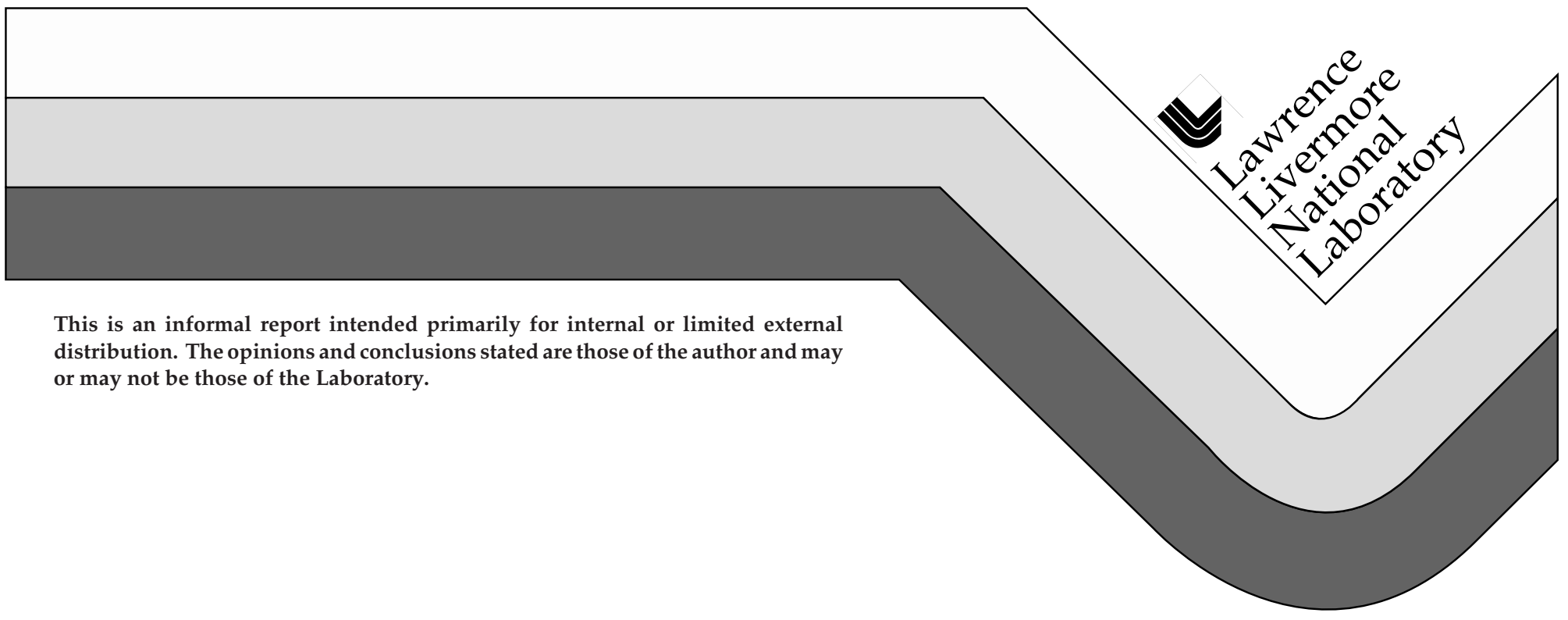




\section{DISCLAIMER}

This document was prepared as an account of work sponsored by an agency of the United States Government. Neither the United States Government nor the University of California nor any of their employees, makes any warranty, express or implied, or assumes any legal liability or responsibility for the accuracy, completeness, or usefulness of any information, apparatus, product, or process disclosed, or represents that its use would not infringe privately owned rights. Reference herein to any specific commercial product, process, or service by trade name, trademark, manufacturer, or otherwise, does not necessarily constitute or imply its endorsement, recommendation, or favoring by the United States Government or the University of California. The views and opinions of authors expressed herein do not necessarily state or reflect those of the United States Government or the University of California, and shall not be used for advertising or product endorsement purposes.

This report has been reproduced directly from the best available copy.

Available to DOE and DOE contractors from the Office of Scientific and Technical Information P.O. Box 62, Oak Ridge, TN 37831

Prices available from (615) 576-8401, FTS 626-8401

Available to the public from the National Technical Information Service

U.S. Department of Commerce 5285 Port Royal Rd. Springfield, VA 22161 


\section{Maximum Likelihood Estimation with Poisson (Counting) Statistics \\ for Waste Drum Inspection*}

\section{Dennis Goodman}

\section{Introduction.}

This note is a preliminary look at the issues involved in waste drum inspection when emission levels are so low that central limit theorem arguments do not apply and counting statistics, rather than the usual Gaussian assumption, must be considered. At very high count rates the assumption of Gaussian statistics is reasonable, and the maximum likelihood arguments that we discuss below for low count rates would lead to the usual approach of least squares fits. Least squares is not the the best technique for low counts, and we will develop the maximum likelihood estimators for the low count case. The usual statistical model for counting problems is the Poisson distribution where the probability of $n$ counts is given by

$$
\operatorname{Pr}[n]=\frac{1}{n !} z^{n} \exp \{-z\}
$$

The parameter $z$ is the mean number of counts: if $N$ has the distribution shown in Eq. 1, then

$$
E\{N\}=z
$$

and

$$
E\left\{N^{2}\right\}=z^{2}+z
$$

Hence, the variance of $N$ is

$$
\sigma_{N}^{2}=E\left\{N^{2}\right\}-[E\{N\}]^{2}=z
$$

Unlike for the Gaussian model, in this case the variance of the observation depends on its intensity. An alternative approach to the one we present here is a variance stabilizing

* Work performed under auspices of the U.S. Department of Energy by the Lawrence Livermore National Laboratory under contract No. W-7405-ENG-48. 
transformation of the data that gets rid of this problem (see Ref. 1). This would possibly allow use of existing least-squares software.

For the particular problem we are considering, two measurements are taken at each detector, say the $k^{t h}$. The first is meant to be an observation of background radiation counts; the second is an observation of activity radiation counts. We denote the counts observed in the first measurement by the random variable $M_{k}$, and the counts in the second measurement by $N_{k}$. The first measurement is taken to account for background effects in the second measurement as it is impossible to measure activity counts alone. Complicating the problem further is the fact that some activity counts occur in the first measurement as well. Each detector measures the counts in spectral bins. It is assumed that the bins used for the first measurement are disjoint from the bins used for the second measurement. This allows us to assume that the two measurements are statistically independent. This is only an approximation, but it permits a reasonably simple model. The mean total background count at the $k^{t h}$ detector is denoted by $w_{k}$. This total is split between the first measurement and the second measurement. For some $0<\tau_{1}<1$ we assume that the mean background count for the first measurement is $\tau_{1} w_{k}$ and the mean background count for the second measurement is $\tau_{2} w_{k}$ where $\tau_{2}=1-\tau_{1}$.

The mean activity count at the $k^{t h}$ detector is denoted by $z_{k}$. The activity counts depend on the location of the activity in the drum. Let $\mathbf{x}$ be a $T$-vector representing the unknown activities in the $T$ voxels. Then we write $z_{k}(\mathbf{x})$ to denote this dependence, which is assumed to be of the form $\mathbf{z}=\mathbf{A x}$, where $\mathbf{A}$ is a $K \times T$ matrix. Some small fraction of the activity counts appear in the first measurement; we denote the mean of this contribution by $\tau_{3} z_{k}(\mathbf{x})$, where $0<\tau_{3} \ll 1$. The mean activity count in the second measurement is $\tau_{4} z_{k}(\mathbf{x})$ where $\tau_{4}=1-\tau_{3}$.

It follows that the mean total count in the first measurement is

$$
E\left\{M_{k}\right\}=\tau_{1} w_{k}+\tau_{3} z_{k}(\mathbf{x})
$$

and the mean total count in the second measurement is

$$
E\left\{N_{k}\right\}=\tau_{2} w_{k}+\tau_{4} z_{k}(\mathbf{x})
$$


where $E$ denotes expected value. Because these two Poisson random variables are independent, their joint density is the product of their marginal densities, giving

$$
\begin{aligned}
\operatorname{Pr}\left(m_{k}, n_{k}, w_{k}, \mathbf{x}\right)= & \frac{1}{m_{k} !}\left[\tau_{1} w_{k}+\tau_{3} z_{k}(\mathbf{x})\right]^{m_{k}} \exp \left\{-\left[\tau_{1} w_{k}+\tau_{3} z_{k}(\mathbf{x})\right]\right\} \\
& \times \frac{1}{n_{k} !}\left[\tau_{2} w_{k}+\tau_{4} z_{k}(\mathbf{x})\right]^{n_{k}} \exp \left\{-\left[\tau_{2} w_{k}+\tau_{4} z_{k}(\mathbf{x})\right]\right\}
\end{aligned}
$$

Rearranging terms gives

$$
\begin{aligned}
\operatorname{Pr}\left(m_{k}, n_{k}, w_{k}, \mathbf{x}\right) & =\frac{1}{m_{k} ! n_{k} !} \exp \left\{-w_{k}-z_{k}(\mathbf{x})\right\} \\
& \times\left[\tau_{1} w_{k}+\tau_{3} z_{k}(\mathbf{x})\right]^{m_{k}}\left[\tau_{2} w_{k}+\tau_{4} z_{k}(\mathbf{x})\right]^{n_{k}}
\end{aligned}
$$

Now let $\mathbf{m}=\left(m_{1}, m_{2}, \ldots, m_{K}\right)$, and similarly for $\mathbf{n}$ and $\mathbf{w}$, and assume again that conditioned on $\mathbf{x}$ the measurements on all of the detectors are independent. Then the joint probability density function of the measurements is:

$$
\begin{aligned}
\operatorname{Pr}(\mathbf{m}, \mathbf{n}, \mathbf{w}, \mathbf{x})=\prod_{k=1}^{K} & \left\{\frac{1}{m_{k} ! n_{k} !} \exp \left\{-w_{k}-z_{k}(\mathbf{x})\right\}\right. \\
& \left.\times\left[\tau_{1} w_{k}+\tau_{3} z_{k}(\mathbf{x})\right]^{m_{k}}\left[\tau_{2} w_{k}+\tau_{4} z_{k}(\mathbf{x})\right]^{n_{k}}\right\}
\end{aligned}
$$

This equation describes the statistical relationship between the unknowns, w and $\mathbf{x}$, and the observed data, $\mathbf{m}$ and $\mathbf{n}$. At this point it should be obvious that accurate values for $\tau_{1}$ and $\tau_{3}$ are crucial to accurate estimates of activity.

\section{Maximum Likelihood Estimation.}

Our problem is to estimate $\mathbf{w}$ and $\mathbf{x}$ given that $\mathbf{m}$ and $\mathbf{n}$ were observed. In other words, we require a function, $g(\mathbf{m}, \mathbf{n})$, called an estimator, of the observables, $\mathbf{m}$ and $\mathbf{n}$, that gives an estimate, $[\hat{\mathbf{w}}, \hat{\mathbf{x}}]$, of the unknowns:

$$
[\hat{\mathbf{w}}, \hat{\mathbf{x}}]=g(\mathbf{m}, \mathbf{n})
$$

In this note, we follow a non-Bayesian approach to estimation; in other words, we are unwilling to assign prior probabilities to the unknowns, and $\mathbf{w}$ and $\mathbf{x}$ are taken to be deterministic, but unknown, vectors. There are a variety of approaches to estimation; one estimator that has a lot of nice asymptotic properties is the maximum likelihood method 
in which the estimate is obtained by substituting the values for the observed counts into Eq. (8), and maximizing with respect to $\mathbf{w}$ and $\mathbf{x}$. As is often the case, it is easier to maximize the log-likelihood function: the logarithm of Eq. (8) is:

$\sum_{k=1}^{K}\left\{-w_{k}-z_{k}(\mathbf{x})+m_{k} \log \left[\tau_{1} w_{k}+\tau_{3} z_{k}(\mathbf{x})\right]+n_{k} \log \left[\tau_{2} w_{k}+\tau_{4} z_{k}(\mathbf{x})\right]\right\}-\sum_{k=1}^{K} \log \left[m_{k} ! n_{k} !\right]$.

The second sum in this equation does not depend on $\mathbf{w}$ and $\mathbf{x}$, so the maximum likelihood estimate is:

$[\hat{\mathbf{w}}, \hat{\mathbf{x}}]=\underset{\mathbf{w} \geq \mathbf{x}}{\operatorname{Argmax}}\left\{\sum_{k=1}^{K}\left\{-w_{k}-z_{k}(\mathbf{x})+m_{k} \log \left[\tau_{1} w_{k}+\tau_{3} z_{k}(\mathbf{x})\right]+n_{k} \log \left[\tau_{2} w_{k}+\tau_{4} z_{k}(\mathbf{x})\right]\right\}\right\}$.

The expected counts must be nonnegative, hence we constrain $\mathbf{w} \geq \mathbf{0}$ and $\mathbf{x} \in S$ where the set $S$ should constrain $\mathbf{z} \geq \mathbf{0}$ and also include any other prior constraints on $\mathbf{x}$. The geometry of the problem should be such that forcing $\mathbf{x}$ to be nonnegative will also make $\mathbf{z}$ nonnegative. This problem is appropriate for solution with the nonlinear version of CCG that uses the enhanced line search described in Section VI.

\section{The Cramér-Rao Inequality.}

The Cramér-Rao inequality is a lower bound on the mean-square estimation error of any unbiased estimator. In other words, it is the best performance one could hope for from an unbiased estimator. Furthermore, for high signal-to-noise ratios the Cramér-Rao inequality is a "tight" bound, and shows what performance is possible. The behavior of this bound is best described asymptotically. Under certain regularity assumptions (which should apply to our problem), it is possible to show that in the limit the maximum likelihood estimator is consistent (becomes unbiased) and is efficient (attains the Cramér-Rao bound). This bound is a useful tool in estimating error variance for the drum problem. We will give a description of this bound for a general Poisson problem; then we apply it to our specific case. Suppose we make $L$ independent observations of counting processes that are parameterized by the unknown vector $\Theta$. The joint PDF is

$$
\operatorname{Pr}\left[\left(n_{1}, n_{2}, \ldots, n_{L}\right) \mid \Theta\right]=\prod_{l=1}^{L} \frac{1}{n_{l} !}\left[\lambda_{l}(\Theta)\right]^{n_{l}} \exp \left\{-\lambda_{l}(\Theta)\right\}
$$


where $\lambda(\Theta)$ is the mean count for the $l^{\text {th }}$ observation parameterized by the vector of unknowns, $\Theta$. We use the symbol $\boldsymbol{\Sigma}$ to denote the error covariance matrix of the given estimator, i.e.,

$$
\boldsymbol{\Sigma}=E\left\{[\hat{\Theta}-\Theta][\hat{\Theta}-\Theta]^{\prime}\right\}
$$

The Cramér-Rao inequality states that if $g(\cdot)$ is unbiased for all values of $\Theta$, then

$$
\boldsymbol{\Sigma} \geq \mathbf{J}^{-1}
$$

where the inequality means that $\boldsymbol{\Sigma}-\mathbf{J}^{-1}$ is positive semi-definite, and $\mathbf{J}$ is the information matrix

$$
\mathbf{J}_{i j}=E\left\{-\frac{\partial^{2}}{\partial \theta_{i} \partial \theta_{j}} \log \operatorname{Pr}\left[\left(n_{1}, n_{2}, \ldots, n_{L}\right) \mid \Theta\right]\right\} .
$$

A consequence of Eq. (14) is that the error variance of $\hat{\theta}_{i}$ must be greater than $\left[\mathbf{J}^{-1}\right]_{i i}$. Those familiar with the Cramér-Rao inequality may be concerned that the version given here is stated in terms of a probability function for a discrete random variable rather than in the usual terms of a probability density function for a continuous random variable. However, both this version and the usual one are consequences of a general theorem that is stated in terms of measure theory and that can be found in advanced texts.

To compute the performance bound we need the following identities which are an immediate consequence of the Taylor series expansion of the exponential:

$$
\begin{gathered}
\sum_{n=0}^{\infty} \frac{e^{-\alpha} \alpha^{n}}{n !}=1 \\
\sum_{n=0}^{\infty} n \frac{e^{-\alpha} \alpha^{n}}{n !}=\alpha
\end{gathered}
$$

Computing the first partials of the logarithm of Eq. (12) gives:

$$
\frac{\partial}{\partial \theta_{i}} \log \operatorname{Pr}\left[\left(n_{1}, n_{2}, \ldots, n_{L}\right) \mid \hat{\Theta}\right]=\sum_{k=1}^{L}\left[-\frac{\partial \lambda_{k}(\Theta)}{\partial \theta_{i}}+\frac{n_{k}}{\lambda_{k}(\Theta)} \frac{\partial \lambda_{k}(\Theta)}{\partial \theta_{i}}\right] .
$$

Computing the second partials and multiplying by -1 gives

$$
\begin{aligned}
& -\frac{\partial^{2}}{\partial \theta_{i} \partial \theta_{j}} \log \operatorname{Pr}\left[\left(n_{1}, n_{2}, \ldots, n_{L}\right) \mid \hat{\Theta}\right]= \\
& \quad \sum_{k=1}^{L}\left[\frac{\partial^{2} \lambda_{k}(\Theta)}{\partial \theta_{i} \partial \theta_{j}}+\frac{n_{k}}{\left[\lambda_{k}(\Theta)\right]^{2}} \frac{\partial \lambda_{k}(\Theta)}{\partial \theta_{i}} \frac{\partial \lambda_{k}(\Theta)}{\partial \theta_{j}}-\frac{n_{k}}{\lambda_{k}(\Theta)} \frac{\partial^{2} \lambda_{k}(\Theta)}{\partial \theta_{i} \partial \theta_{j}}\right] .
\end{aligned}
$$


The entries of the information matrix are obtained by computing the expected value of Eq. (18). To simplify the algebra, note that the $k^{\text {th }}$ term of the right hand side of Eq. (18) is a function only of $\Theta$ and $n_{k}$ and so may be written temporarily as $f_{k}\left(\Theta, n_{k}\right)$. Using this notation and Eq. (12) gives

$$
\begin{aligned}
\mathbf{J}_{i j} & =\sum_{n_{1}=0}^{\infty} \cdots \sum_{n_{L}=0}^{\infty}\left[\sum_{k=1}^{L} f_{k}\left(\Theta, n_{k}\right)\right]\left[\prod_{l=1}^{L} \frac{e^{-\lambda_{l}(\Theta)}\left[\lambda_{l}(\Theta)\right]^{n_{l}}}{n_{l} !}\right] \\
& =\sum_{k=1}^{L} \sum_{n_{1}=0}^{\infty} \cdots \sum_{n_{L}=0}^{\infty}\left[f_{k}\left(\Theta, n_{k}\right) \prod_{l=1}^{L} \frac{e^{-\lambda_{l}(\Theta)}\left[\lambda_{l}(\Theta)\right]^{n_{l}}}{n_{l} !}\right] \\
& =\sum_{k=1}^{L}\left[\sum_{n_{k}=0}^{\infty} f_{k}\left(\Theta, n_{k}\right) \frac{e^{-\lambda_{k}(\Theta)}\left[\lambda_{k}(\Theta)\right]^{n_{k}}}{n_{k} !}\right]
\end{aligned}
$$

where the infinite sums were eliminated by repeatedly applying Eq. (16a). The remaining infinite sum is eliminated by substituting the $k^{\text {th }}$ term of Eq. (18) for $f_{k}\left(\Theta, n_{k}\right)$ and applying Eqs. (16a) and (16b) to give

$$
\mathbf{J}_{i j}=\sum_{l=1}^{L} \frac{1}{\lambda_{l}(\Theta)} \frac{\partial \lambda_{l}(\Theta)}{\partial \theta_{i}} \frac{\partial \lambda_{l}(\Theta)}{\partial \theta_{j}} .
$$

To gain some intuition, note that the inverse of $\mathbf{J}$ is the variance bound; therefore, good optical systems will have a "large" J. In particular, Eq. (15) shows that to make $\mathbf{J}$ big, the number of photons striking each detector should be made to vary as much as possible with changing $\Theta$. We now apply Eq. (20) to the joint probability function of Eq. (8). There are $2 K$ independent measurements; i.e., $m_{k}$ and $n_{k}$ at each of the $K$ detectors. Eq. (5) gives the means of these measurements parameterized by the unknowns, $\mathbf{w}$ and $\mathbf{x}$. Let $\theta_{i}, \theta_{j}$ each represent arbitrary parameters; i.e., some $w_{r}$ or $x_{s}$. Then:

$$
\begin{aligned}
\mathbf{J}_{i j}=\sum_{k=1}^{K} & \left\{\frac{1}{\left[\tau_{1} w_{k}+\tau_{3} z_{k}(\mathbf{x})\right]} \frac{\partial\left[\tau_{1} w_{k}+\tau_{3} z_{k}(\mathbf{x})\right]}{\partial \theta_{i}} \frac{\partial\left[\tau_{1} w_{k}+\tau_{3} z_{k}(\mathbf{x})\right]}{\partial \theta_{j}}\right. \\
& \left.+\frac{1}{\left[\tau_{2} w_{k}+\tau_{4} z_{k}(\mathbf{x})\right]} \frac{\partial\left[\tau_{2} w_{k}+\tau_{4} z_{k}(\mathbf{x})\right]}{\partial \theta_{i}} \frac{\partial\left[\tau_{2} w_{k}+\tau_{4} z_{k}(\mathbf{x})\right]}{\partial \theta_{j}}\right\} .
\end{aligned}
$$

If $\theta_{i}=\theta_{j}=w_{r}$, then

$$
\mathbf{J}_{i i}=\frac{\tau_{1}^{2}}{\left[\tau_{1} w_{k}+\tau_{3} z_{k}(\mathbf{x})\right]}+\frac{\tau_{2}^{2}}{\left[\tau_{2} w_{k}+\tau_{4} z_{k}(\mathbf{x})\right]}
$$


If $\theta_{i}=w_{r}$ and $\theta_{j}=w_{s}$ with $r \neq s$ then

$$
\mathbf{J}_{i j}=0 \text {. }
$$

If $\theta_{i}=w_{r}$ and $\theta_{j}=x_{s}$ then

$$
\mathbf{J}_{i j}=\left[\frac{\tau_{1} \tau_{3}}{\left[\tau_{1} w_{r}+\tau_{3} \mathbf{z}_{r}(\mathbf{x})\right]}+\frac{\tau_{2} \tau_{4}}{\left[\tau_{2} w_{r}+\tau_{4} \mathbf{z}_{r}(\mathbf{x})\right]}\right] \frac{\left.\partial z_{r}(\mathbf{x})\right]}{\partial x_{s}} .
$$

Finally, if $\theta_{i}=x_{r}$ and $\theta_{j}=x_{s}$ then

$$
\mathbf{J}_{i j}=\sum_{k=1}^{K}\left[\frac{\tau_{3}^{2}}{\left[\tau_{1} w_{k}+\tau_{3} z_{k}(\mathbf{x})\right]}+\frac{\tau_{4}^{2}}{\left[\tau_{2} w_{k}+\tau_{4} z_{k}(\mathbf{x})\right]}\right] \frac{\partial z_{k}(\mathbf{x})}{\partial x_{r}} \frac{\partial z_{k}(\mathbf{x})}{\partial x_{s}}
$$

These equations can be simplified further. Recalling that the relationship between $\mathbf{z}$ and $\mathbf{x}$ is $\mathbf{z}=\mathbf{A x}$, we see that

$$
\frac{\partial z_{k}(\mathbf{x})}{\partial x_{t}}=a_{k t}=[\mathbf{A}]_{k t}
$$

Eq. (22) gives all of the elements of the information matrix $\mathbf{J}$. Note that $\mathbf{J}$ depends on the unknowns, not the counts. Therefore, the unknowns must be estimated first, so $\mathbf{J}$ itself is determined from the data. What we really want is the total amount of activity, which is

$$
u=\sum_{t=1}^{T} x_{t}
$$

The variance bound on $u$ can be obtained from $\mathbf{J}$. If $\Theta^{\prime}=\left[\mathbf{w}^{\prime}, \mathbf{x}^{\prime}\right]^{\prime}$ is a vector of the unknowns, and $\mathbf{d}^{\prime}=\left[\mathbf{0}^{\prime}, \mathbf{1}^{\prime}\right]^{\prime}$ where $\mathbf{0}$ is a K-vector of zeroes and $\mathbf{1}$ is a T-vector of ones, then

$$
\begin{gathered}
u=\mathbf{d}^{\prime} \Theta, \\
\sigma_{u}^{2} \geq \mathbf{d}^{\prime} \mathbf{J}^{-1} \mathbf{d} .
\end{gathered}
$$

This equation is important for a couple of reasons. The first is that it allows us to estimate the accuracy of a particular experiment. The second is that it allows us to consider the issue of experiment design: we should design our experiments to minimize the right hand side of this equation. This allows us to design our equipment for best results without 
resorting to hours of Monte-Carlo runs. It is not necessary to completely compute the inverse of $\mathbf{J}$ to get the variance bound for $u$. If we solve the set of linear equations

$$
\mathbf{J b}=\mathbf{d}
$$

with an appropriate iterative scheme, then

$$
\mathbf{d}^{\prime} \mathbf{J}^{-1} \mathbf{d}=\mathbf{b}^{\prime} \mathbf{J} \mathbf{b}
$$

Its important to note once again that this bound is "tight" only at high count rates. As the rates decline, the bound will typically exhibit a threshold effect: below some count rate the actual variance will be much larger than the bound. At lower rates, a bootstrap technique is probably appropriate (see Ref. 6). This method is a lot more costly than what I have outlined above, but it is the only option at low counting rates.

We conclude this section with an example of how the Cramér-Rao inequality can be used in design and in performance calculations. First, we write $\mathbf{J}$ as the block matrix

$$
\mathbf{J}=\left[\begin{array}{ll}
\mathbf{J}_{\mathbf{w w}} & \mathbf{J}_{\mathbf{w x}} \\
\mathbf{J}_{\mathbf{w x}}^{\prime} & \mathbf{J}_{\mathbf{x x}}
\end{array}\right],
$$

Where $\mathbf{J}_{\mathbf{w} \mathbf{w}}$ corresponds to the terms of Eq. (22a), $\mathbf{J}_{\mathbf{w} \mathbf{x}}$ corresponds to the terms of Eq. (22b), and $\mathbf{J}_{\mathbf{x} \mathbf{x}}$ corresponds to the terms of Eq. (22c). The inverse of this matrix is

$$
\mathbf{R}=\left[\begin{array}{ll}
\mathbf{R}_{\mathrm{ww}} & \mathbf{R}_{\mathrm{wx}} \\
\mathbf{R}_{\mathrm{wx}}^{\prime} & \mathbf{R}_{\mathbf{x x}}
\end{array}\right],
$$

A matrix identity on pp. 217-218 of Ref. 2 gives:

$$
\mathbf{R}_{\mathbf{x x}}=\left[\mathbf{J}_{\mathbf{x x}}-\mathbf{J}_{\mathbf{w} \mathbf{x}}^{\prime} \mathbf{J}_{\mathbf{w} \mathbf{w}}^{-1} \mathbf{J}_{\mathbf{w x}}\right]^{-1}
$$

Thus, Eq. (24b) becomes

$$
\sigma_{u}^{2} \geq \mathbf{1}^{\prime} \mathbf{R}_{\mathbf{x x}} \mathbf{1}
$$

Now, suppose we are interested in the effect of integration time on the accuracy of our measurements. The effect of changing integration time by some factor $\delta$ is to change the mean counts by the same factor. In particular, we replace $w_{k}$ and $z_{k}$ by, respectively, $\delta w_{k}$ and $\delta z_{k}$ in all of our equations. A consequence of Eq. (22) is that $\mathbf{J}_{\mathbf{w w}}, \mathbf{J}_{\mathbf{w x}}$, and $\mathbf{J}_{\mathbf{x x}}$ 
should be replaced by, respectively, $\delta^{-1} \mathbf{J}_{\mathbf{w w}}, \mathbf{J}_{\mathbf{w x}}$, and $\delta \mathbf{J}_{\mathbf{x x}}$. Now substituting into Eq. (29) shows that Eq. (30) becomes

$$
\sigma_{u}^{2} \geq \delta^{-1} \mathbf{1}^{\prime} \mathbf{R}_{\mathbf{x x}} \mathbf{1}
$$

i.e., the error variance of our estimate is inversely proportional to integration time.

\section{Derivatives of the Log-Likelihood Function.}

Any optimization algorithm that we might want to use (CCG, L-BFGS, etc.) will need the first derivatives of the function being maximized in Eq. (11). We give them here.

$$
\begin{aligned}
\frac{\partial}{\partial w_{k}} & =-1+\frac{\tau_{1} m_{k}}{\left[\tau_{1} w_{k}+\tau_{3} z_{k}(\mathbf{x})\right]}+\frac{\tau_{2} n_{k}}{\left[\tau_{2} w_{k}+\tau_{4} z_{k}(\mathbf{x})\right]} \\
\frac{\partial}{\partial x_{t}} & =\sum_{k=1}^{K}\left[-1+\frac{\tau_{3} m_{k}}{\left[\tau_{1} w_{k}+\tau_{3} z_{k}(\mathbf{x})\right]}+\frac{\tau_{4} n_{k}}{\left[\tau_{2} w_{k}+\tau_{4} z_{k}(\mathbf{x})\right]}\right] \frac{\partial z_{k}(\mathbf{x})}{\partial x_{t}}
\end{aligned}
$$

Eq. (27b) can be written in matrix form. Let $r(k)$ represent the $k^{t h}$ term in the large brackets. Then we may write the vector of first partials with respect to $\mathbf{x}$ as

$$
\nabla_{\mathbf{x}}=\mathbf{A}^{\prime} \mathbf{r}
$$

\section{One Approach to the Solution.}

It might make more sense to model $\mathbf{w}$ as a function of whatever else is in the drum and estimate this voxel density as well, i.e. write $w_{k}(\mathbf{y})$ where $\mathbf{y}$ is a vector representing the voxel densities of whatever gave rise to the background. However, for the moment we will not assume a functional relationship: instead, we estimate the mean background counts in each detector directly. We could apply CCG or some other algorithm directly to Eq. (11), but the background measurement $w_{k}$ appears in only the $k^{\text {th }}$ term, and an explicit solution in terms of $\mathbf{x}$ and the observables is possible; i.e., the maximizing value of $w_{k}$ can be solved for directly. This greatly reduces the number of variables that must be solved for by CCG as it is only necessary to optimize over $\mathbf{x}$. The derivative of Eq. (11) wrt $w_{k}$ is given by Eq. (32a). The second derivative is:

$$
\frac{\partial^{2}}{\partial w_{k}^{2}}=-\frac{\tau_{1}^{2} m_{k}}{\left[\tau_{1} w_{k}+\tau_{3} z_{k}(\mathbf{x})\right]^{2}}-\frac{\tau_{2}^{2} n_{k}}{\left[\tau_{2} w_{k}+\tau_{4} z_{k}(\mathbf{x})\right]^{2}}
$$


Assuming that $m_{k}, n_{k}$, and $z_{k}(\mathbf{x})$ are all nonnegative, Eq. (33) shows that the second derivative is negative for any $w_{k}>0$. Hence, Eq. (11) is a concave function of $w_{k}$ in the region $w_{k} \geq 0$, and any value of $w_{k}$ that makes the first derivative zero in this region will also be the global maximizer of Eq. (11) wrt $w_{k}$ in this region. To obtain this maximum we set Eq. (32a) to zero and compute the solutions, which are

$$
w_{k}=\frac{\left(-\tau_{1} \tau_{4}-\tau_{2} \tau_{3}\right) z_{k}(\mathbf{x})+\tau_{1} \tau_{2}\left(m_{k}+n_{k}\right)_{-}^{+} f_{k}\left(z_{k}(\mathbf{x})\right)}{2 \tau_{1} \tau_{2}}
$$

where

$$
f_{k}\left(z_{k}(\mathbf{x})\right)=\sqrt{\tau_{1}^{2} \tau_{2}^{2}\left(m_{k}+n_{k}\right)^{2}+2 \tau_{1} \tau_{2}\left(\tau_{1}-\tau_{3}\right)\left(m_{k}-n_{k}\right) z_{k}(\mathbf{x})+\left(\tau_{1}-\tau_{3}\right)^{2} z_{k}(\mathbf{x})^{2}} .
$$

Fig. 1 is a plot if Eq. (32a) as a function of $w_{k}$. The points where the graph intersects the $w$ axis are given by Eq. (34). Assuming that $\tau_{4} / \tau_{2}>\tau_{3} / \tau_{1}$, the two vertical asymptotes shown in this figure are $a=-\tau_{3} / \tau_{1}$ and $b=-\tau_{4} / \tau_{2}$. We conclude that there is no problem with complex roots and that only the solution with the positive square root could possibly give a nonnegative $w_{k}$. If there is no zero first derivative for $w_{k}>0$, then the global maximizer in the region $w_{k} \geq 0$ is $w_{k}=0$.

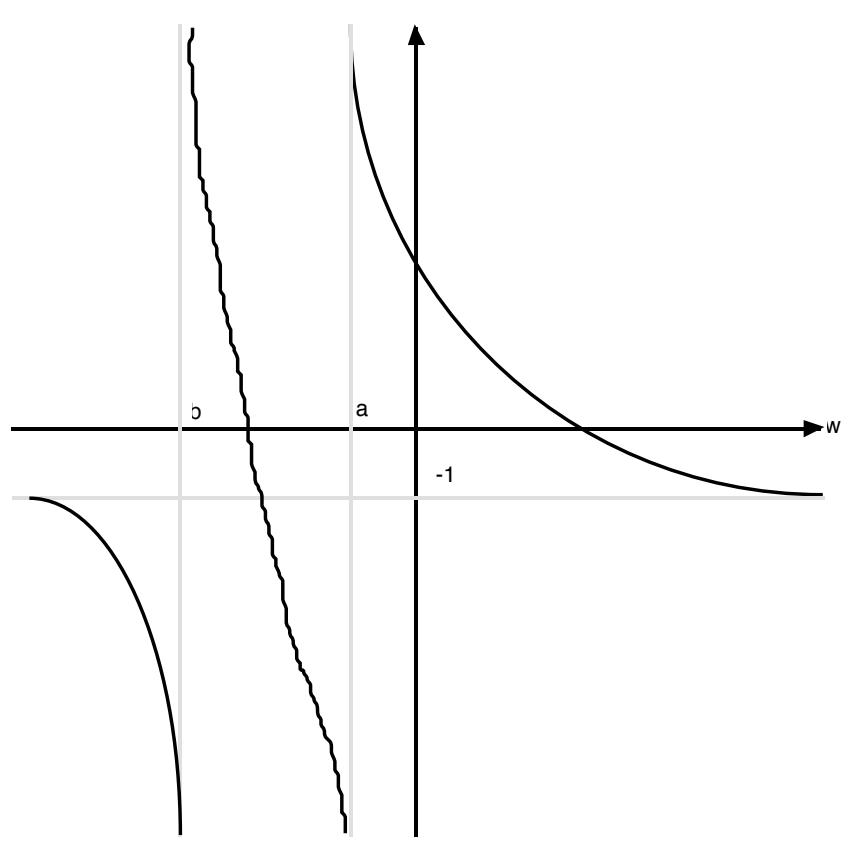

Fig. 1. Plot of Eq. (32a) 
In order to evaluate Eq. (11) in terms of x, Eqs. (34-35) are used to determine w, any negative values are set to zero, and the result, which we denote by $\tilde{\mathbf{w}}$, is substituted into Eq. (11). However, calculating the derivatives with respect to $\mathbf{x}$ must take into account the dependence of the optimal values of $\mathbf{w}$ on $\mathbf{z}$. In particular, let the $k^{\text {th }}$ term of Eq. (11) be written as

$$
s_{k}\left[z_{k}(\mathbf{x})\right]=h_{k}\left\{z_{k}(\mathbf{x}), \tilde{w}_{k}\left[z_{k}(\mathbf{x})\right]\right\} .
$$

Then Eq. (11) becomes

$$
\hat{\mathbf{x}}=\underset{\mathbf{x} \in S}{\operatorname{Argmax}}\{L(\mathbf{x})\}
$$

where

$$
L(\mathbf{x})=\sum_{k=1}^{K} s_{k}\left[z_{k}(\mathbf{x})\right]
$$

We will at times suppress the dependence of $\tilde{w}_{k}$ on $z_{k}(\mathbf{x})$ for notational convenience. The derivatives with respect to $\mathbf{x}$ of the function being maximized are:

$$
\begin{aligned}
\frac{\partial L(\mathbf{x})}{\partial x_{t}} & =\sum_{k=1}^{K} \frac{\partial s_{k}\left[z_{k}(\mathbf{x})\right]}{\partial z_{k}} \frac{\partial z_{k}(\mathbf{x})}{\partial x_{t}} \\
& =\sum_{k=1}^{K} \frac{\partial s_{k}\left[z_{k}(\mathbf{x})\right]}{\partial z_{k}} a_{k t}
\end{aligned}
$$

where

$$
\begin{gathered}
\frac{\partial s_{k}\left[z_{k}(\mathbf{x})\right]}{\partial z_{k}}=\frac{\partial h_{k}\left\{z_{k}(\mathbf{x}), \tilde{w}_{k}\right\}}{\partial z_{k}}+\frac{\partial h_{k}\left\{z_{k}(\mathbf{x}), \tilde{w}_{k}\right\}}{\partial w_{k}} \frac{\partial \tilde{w}_{k}\left[z_{k}(\mathbf{x})\right]}{\partial z_{k}} \\
\frac{\partial h_{k}\left\{z_{k}(\mathbf{x}), \tilde{w}_{k}\right\}}{\partial z_{k}}=-1+\frac{\tau_{3} m_{k}}{\left[\tau_{1} \tilde{w}_{k}+\tau_{3} z_{k}(\mathbf{x})\right]}+\frac{\tau_{4} n_{k}}{\left[\tau_{2} \tilde{w}_{k}+\tau_{4} z_{k}(\mathbf{x})\right]}, \\
\frac{\partial h_{k}\left\{z_{k}(\mathbf{x}), \tilde{w}_{k}\right\}}{\partial w_{k}}=-1+\frac{\tau_{1} m_{k}}{\left[\tau_{1} \tilde{w}_{k}+\tau_{3} z_{k}(\mathbf{x})\right]}+\frac{\tau_{2} n_{k}}{\left[\tau_{2} \tilde{w}_{k}+\tau_{4} z_{k}(\mathbf{x})\right]},
\end{gathered}
$$

and

$$
\frac{\partial \tilde{w}_{k}\left[z_{k}(\mathbf{x})\right]}{\partial z_{k}}=\frac{-\tau_{1} \tau_{4}-\tau_{2} \tau_{3}}{2 \tau_{1} \tau_{2}}+\frac{\tau_{1} \tau_{2}\left(\tau_{1}-\tau_{3}\right)\left(m_{k}-n_{k}\right)+\left(\tau_{1}-\tau_{3}\right)^{2} z_{k}(\mathbf{x})}{2 \tau_{1} \tau_{2} f_{k}\left(z_{k}(\mathbf{x})\right)}
$$

Where Eq. (37e) is set to zero if $w_{k}$ calculated in Eq. (34) is not positive.

If we define $\mathbf{r}(\mathbf{x})$ to be the vector whose $k^{\text {th }}$ element is given by Eq. (37b), then from Eq. (37a) we see that the gradient of $L(\mathbf{x})$ is

$$
\nabla_{\mathbf{x}} L(\mathbf{x})=\mathbf{A}^{\prime} \mathbf{r}(\mathbf{x})
$$


The function and its partial derivatives are sufficient to apply the standard version of CCG and other optimization algorithms. However, the particular nature of this problem, and many other problems, suggests additional modifications. We discuss these modifications in the next section.

\section{Speeding Up the Line Search}

One advantage, among others, that CCG has over other approaches is that a line search is conducted at each iteration. For the general problem, the cost of a function plus gradient evaluation is the same whether it is done at the start of an iteration or during the line search. Therefore, considerable effort is spent on the line search. However, the cost of the line search can be reduced considerably for problems like the one discussed in this note. Most algorithms work as follows: at the $j^{\text {th }}$ iteration we are given the current best estimate, $\hat{\mathbf{x}}^{j}$, and a direction, $\mathbf{d}^{j}$, along which to search for a better estimate, $\hat{\mathbf{x}}^{j+1}$. In particular, the line search involves solving

$$
\alpha^{j+1}=\underset{\alpha>0}{\operatorname{Argmax}}\{L(\mathbf{x}(\alpha))\}
$$

where

$$
L(\mathbf{x}(\alpha))=\sum_{k=1}^{K} s_{k}\left\{z_{k}\left(\hat{\mathbf{x}}^{j}+\alpha \mathbf{d}^{j}\right)\right\} .
$$

Once $\alpha^{j+1}$ has been determined, the next estimate is

$$
\hat{\mathbf{x}}^{j+1}=\hat{\mathbf{x}}^{j}+\alpha^{j+1} \mathbf{d}^{j} .
$$

Note that in Eq. (38b) the $k^{\text {th }}$ term depends only on $z_{k}$ : it is a function of no other $z_{l}$ for $l \neq k$. Consequently, evaluating $s_{k}\left\{z_{k}\right\}$ for $k=1, \ldots, K$ involves only $\mathcal{O}(K)$ floating point operations, and the major effort in evaluating $L(\mathbf{x})$ is the $\mathcal{O}(K T)$ floating point operations involved in computing $\mathbf{z}=\mathbf{A x}$. Now note that if we write $\mathbf{z}$ as a function of the line search parameter $\alpha$ we have:

$$
\begin{aligned}
\mathbf{z}(\alpha) & =\mathbf{A}\left[\hat{\mathbf{x}}^{j}+\alpha \mathbf{d}^{j}\right] \\
& =\mathbf{z}^{j}+\alpha \mathbf{A} \mathbf{d}^{j}
\end{aligned}
$$

If we take advantage of the second line of Eq. (39), will only need to compute one matrix multiplication with $\mathbf{A}$ in evaluating $L(\mathbf{x}(\alpha))$, no matter how many values of $\alpha$ we try. A 
similar situation exists for the directional derivative; i.e., the derivative of $L(\mathbf{x}(\alpha))$ with respect to $\alpha$.

$$
\begin{aligned}
\frac{\partial}{\alpha}\left\{\sum_{k=1}^{K} s_{k}\left\{z_{k}\left(\hat{\mathbf{x}}^{j}+\alpha \mathbf{d}^{j}\right)\right\}\right\} & =\sum_{k=1}^{K} \frac{\partial s_{k}}{\partial z_{k}} \times \frac{\partial}{\partial \alpha}\left\{\left[\mathbf{A}\left[\hat{\mathbf{x}}^{j}+\alpha \mathbf{d}^{j}\right]\right]_{k}\right\} \\
& =\sum_{k=1}^{K} \frac{\partial s_{k}}{\partial z_{k}} \times\left[\mathbf{A d}^{j}\right]_{k} \\
& =\mathbf{r}(\mathbf{x})^{\prime} \mathbf{A} \mathbf{d}^{j} \\
& =\left[\nabla_{\mathbf{x}} L(\mathbf{x})\right]^{\prime} \mathbf{d}^{j}
\end{aligned}
$$

The last line of Eq. (40) shows that the directional derivative is the dot product of the gradient with the direction; this is the calculation made by standard algorithms. Note, however, the next to last line of this equation: $\mathbf{r}(\mathbf{x})$ depends on $\alpha$, but $\mathbf{A d}^{j}$ does not. Once again we have shown that there need be only one matrix multiplication during a line search iteration. We have not told the whole story. For the bending line search used in $\mathrm{CCG}, \mathbf{d}$ is only a piecewise constant function of $\alpha$ : each time a given $x_{t}$ hits a constraint, the corresponding $d_{t}$ is set to zero. Because only one element of $\mathbf{d}$ changes, a complete recalculation of $\mathbf{A d} \mathbf{d}^{j}$ is not necessary. The subroutines MATCOL and UPDATE account for the changes by multiplying only the $t^{t h}$ column of $\mathbf{A}$ and making the appropriate changes in Eqs. (38-40).

An additional reduction in effort can be obtained by computing the second partial with respect to $\alpha$. The second partial is valuable because then a quadratic approximation to $L(\mathbf{x}(\alpha))$ then becomes available, and the $\alpha$ that minimizes this quadratic is a good starting point for the line search. In particular the 1-D Newton's method estimate for the initial $\alpha_{0}$ is

$$
\alpha_{0}=-\frac{\left.\frac{\partial L(\mathbf{x}(\alpha))}{\partial \alpha}\right|_{\alpha=0}}{\left.\frac{\partial^{2} L(\mathbf{x}(\alpha))}{\partial \alpha^{2}}\right|_{\alpha=0}} .
$$

For general problems with many unknowns, direct calculation of second derivative information is impossible, as the matrix of second partials (i.e., the Hessian) must be calculated. However, we may once again take advantage of the particular structure of our problem: 


$$
\begin{aligned}
\frac{\partial^{2}}{\alpha^{2}}\left\{\sum_{k=1}^{K} s_{k}\left\{z_{k}\left(\hat{\mathbf{x}}^{j}+\alpha \mathbf{d}^{j}\right)\right\}\right\} & =\sum_{k=1}^{K} \frac{\partial^{2} s_{k}}{\partial z_{k}^{2}}\left(\frac{\partial}{\partial \alpha}\left\{\left[\mathbf{A}\left[\hat{\mathbf{x}}^{j}+\alpha \mathbf{d}^{j}\right]\right]_{k}\right\}\right)^{2}+\sum_{k=1}^{K} \frac{\partial s_{k}}{\partial z_{k}} \frac{\partial^{2}}{\partial \alpha^{2}}\left\{\left[\mathbf{A}\left[\hat{\mathbf{x}}^{j}+\alpha \mathbf{d}^{j}\right]\right]_{k}\right\} \\
& =\sum_{k=1}^{K} \frac{\partial^{2} s_{k}}{\partial z_{k}^{2}} \times\left(\left[\mathbf{A} \mathbf{d}^{j}\right]_{k}\right)^{2}
\end{aligned}
$$

Because $\mathbf{z}(\alpha)$ is linear in $\alpha$, it has a zero second partial, so the second sum in the first line of Eq. (42) is zero. Once the first partial has been computed, $\mathbf{A d}^{j}$ is available, so most of the additional work involves computing $\partial^{2} s_{k} / \partial z_{k}^{2}$ for $k=1, \ldots, K$, but this takes only $\mathcal{O}(K)$ operations. The equations are messy, however. Recalling the definition of $s_{k}\left(z_{k}\right)$ given in Eq. (36a), we have:

$$
\begin{aligned}
\frac{\partial^{2} s_{k}}{\partial z_{k}^{2}}= & \frac{\partial^{2} h_{k}\left(z_{k}, \tilde{w}_{k}\left(z_{k}\right)\right)}{\partial z_{k}^{2}}+2 \frac{\partial^{2} h_{k}\left(z_{k}, \tilde{w}_{k}\left(z_{k}\right)\right)}{\partial z_{k} \partial w_{k}} \frac{\partial \tilde{w}_{k}\left(z_{k}\right)}{\partial z_{k}} \\
& +\frac{\partial h_{k}\left(z_{k}, \tilde{w}_{k}\left(z_{k}\right)\right)}{\partial w_{k}} \frac{\partial^{2} \tilde{w}_{k}\left(z_{k}\right)}{\partial z_{k}^{2}}+\frac{\partial^{2} h_{k}\left(z_{k}, \tilde{w}_{k}\left(z_{k}\right)\right)}{\partial w_{k}^{2}}\left[\frac{\partial \tilde{w}_{k}\left(z_{k}\right)}{\partial z_{k}}\right]^{2}
\end{aligned}
$$

where

$$
\begin{aligned}
\frac{\partial^{2} h_{k}\left(z_{k}, \tilde{w}_{k}\left(z_{k}\right)\right)}{\partial z_{k}^{2}} & =-\frac{\tau_{3}^{2} m_{k}}{\left[\tau_{1} \tilde{w}_{k}+\tau_{3} z_{k}\right]^{2}}-\frac{\tau_{4}^{2} n_{k}}{\left[\tau_{2} \tilde{w}_{k}+\tau_{4} z_{k}\right]^{2}} \\
\frac{\partial^{2} h_{k}\left(z_{k}, \tilde{w}_{k}\left(z_{k}\right)\right)}{\partial z_{k} \partial w_{k}} & =-\frac{\tau_{1} \tau_{3} m_{k}}{\left[\tau_{1} \tilde{w}_{k}+\tau_{3} z_{k}\right]^{2}}-\frac{\tau_{2} \tau_{4} n_{k}}{\left[\tau_{2} \tilde{w}_{k}+\tau_{4} z_{k}\right]^{2}} \\
\frac{\partial^{2} h_{k}\left(z_{k}, \tilde{w}_{k}\left(z_{k}\right)\right)}{\partial w_{k}^{2}} & =-\frac{\tau_{1}^{2} m_{k}}{\left[\tau_{1} \tilde{w}_{k}+\tau_{3} z_{k}\right]^{2}}-\frac{\tau_{2}^{2} n_{k}}{\left[\tau_{2} \tilde{w}_{k}+\tau_{4} z_{k}\right]^{2}} \\
\frac{\partial^{2} \tilde{w}_{k}\left(z_{k}\right)}{\partial z_{k}^{2}} & =\frac{\left(\tau_{1}-\tau_{3}\right)^{2}}{2 \tau_{1} \tau_{2}}\left(\frac{1}{f_{k}\left(z_{k}\right)}-\frac{\left[\tau_{1} \tau_{2}\left(m_{k}-n_{k}\right)+\left(\tau_{1}-\tau_{3}\right) z_{k}\right]^{2}}{\left[f_{k}\left(z_{k}\right)\right]^{3}}\right)
\end{aligned}
$$

the first partials are given in Eq. (37), and $f_{k}\left(z_{k}\right)$ is defined in Eq. (35). The partials of $\tilde{w}_{k}$ with respect to $z_{k}$ should be set to zero if the value of $w_{k}$ computed by Eq. (34) is not positive.

\section{Action Plan.}

It has been observed that the current estimation algorithm, which is an implementation of the expectation-maximization (EM) algorithm, gives activity estimates that are a function of integration time. The current implementation of the EM algorithm does 
not maximize the likelihood function described above. In particular, at each detector the background observation is subtracted from the activity observation; then maximum likelihood is applied to the result. Furthermore, if the result of the subtraction is a negative count, then the count is arbitrarily set to zero. Consequently, the most likely reason for the dependence on integration time is estimator bias due to an improper likelihood model.

To verify this, the first step should be to implement a simulator based on the model given above. If the EM algorithm is applied to the simulated data and bias is observed, then we know that the current implementation of the EM algorithm is unsatisfactory. On the other hand, if the CCG method described herein is applied to the simulator and there is no bias, but there is bias when it is applied to real data, then the model is incorrect and a new likelihood formulation must be developed for the new model.

I have developed a FORTRAN version of the algorithm described in this paper, and I have tested it on a simulated problem that uses a very simple projector. This problem is not directly related to the waste drum problem, but it did use the first and second measurement model described in Section I. Estimator bias did not appear to be a major issue, but I did not conduct extensive simulations. At this point the code should be converted to $\mathrm{C}$, the projectors appropriate for waste drum inspection should be incorporated, and the code should be extensively tested on real and simulated waste drum data. The fact that bias was not a serious issue on the simple problem is very encouraging for the waste drum problem. Once the estimator codes have been developed, code for the Cramér-Rao bound calculations should be developed and compared to Monte-Carlo runs to verify both the estimation algorithm and the bounds.

\section{References}

1. P. McCullagh and J. A. Nelder, Generalized Linear Models, New York: Chapman and Hall, 1983.

2. J. V. Candy, Signal Processing: The Model-Based Approach, New York: McGrawHill, 1986.

3. D. M. Goodman, E. M. Johansson, and T. W. Lawrence, "On Applying the Conjugate Gradient Algorithm to Image Processing Problems," in Multivariate Analysis: 
Future Directions, C. R. Rao, Editor, Amsterdam: North-Holland, 1993.

4. D. L. Snyder and M. I. Miller, Random Point Processes in Time and Space, New York: Springer-Verlag, 1991.

5. H. L. Van Trees, Detection, Estimation, and Modulation Theory, Part 1, New York: Wiley, 1968.

6. B. Efron, The Jacknife, the Bootstrap, and Other Resampling Plans, Philiadelphia: SIAM, 1982. 


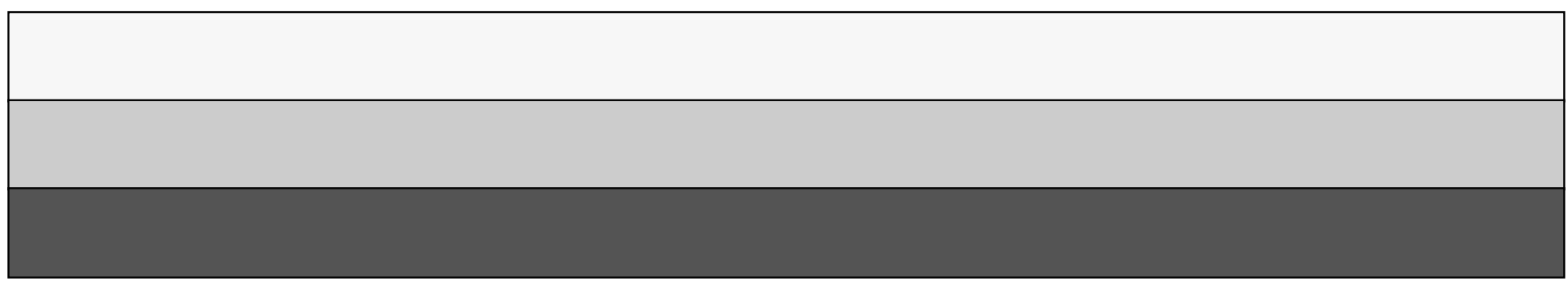

